\title{
Cortical Evolution: Introduction to the Reptilian Cortex
}

\author{
Gilles Laurent, Julien Fournier, Mike Hemberger, Christian Müller, \\ Robert Naumann, Janie M. Ondracek, Lorenz Pammer, Samuel Reiter, \\ Mark Shein-Idelson, Maria Antonietta Tosches, and Tracy Yamawaki
}

\begin{abstract}
Some 320 million years ago (MYA), the evolution of a protective membrane surrounding the embryo, the amnion, enabled vertebrates to develop outside water and thus invade new terrestrial niches. These amniotes were the ancestors of today's mammals and sauropsids (reptiles and birds). Present-day reptiles are a diverse group of more than 10,000 species that comprise the sphenodon, lizards, snakes, turtles and crocodilians. Although turtles were once thought to be the most "primitive" among the reptiles, current genomic data point toward two major groupings: the Squamata (lizards and snakes) and a group comprising both the turtles and the Archosauria (dinosaurs and modern birds and crocodiles). Dinosaurs inhabited the Earth from the Triassic (230 MYA), at a time when the entire landmass formed a single Pangaea. Dinosaurs flourished from the beginning of the Jurassic to the mass extinction at the end of the Cretaceous (65 MYA), and birds are their only survivors. What people generally call reptiles is thus a group defined in part by exclusion: it gathers amniote species that are neither mammals nor birds, making the reptiles technically a paraphyletic grouping. Despite this, the so-defined reptiles share many evolutionary, anatomical, developmental, physiological (e.g., ectothermia), and functional features. It is thus reasonable to talk about a "reptilian brain."
\end{abstract}

\section{Reptilian Brain Structure and Evolution}

The diversity of reptiles and their evolutionary relationship to mammals make reptilian brains great models to explore questions related to the structural and functional evolution of vertebrate neural circuits. To this end, comparative studies

G. Laurent $(\bowtie) \cdot$ J. Fournier $\bullet$ M. Hemberger $\bullet$ C. Müller • R. Naumann • J.M. Ondracek • L. Pammer $\bullet$ S. Reiter $\bullet$ M. Shein-Idelson $\bullet$ M.A. Tosches $\bullet$ T. Yamawaki

Max Planck Institute for Brain Research, Max von Laue Str. 4, 60438 Frankfurt am Main, Germany

e-mail: gilles.laurent@brain.mpg.de 
seek to identify homologies, i.e., structural or molecular similarities due to common ancestry, at a variety of levels, e.g., brain regions, circuits, or cell types. Homologies can be inferred from extant species by using a comparative approach within a phylogenetic framework. Vertebrate brains have been classically compared in terms of morphology, connectivity, and neurochemistry. However, adult neuroanatomy may not be sufficient to determine homologies without ambiguity.

Vertebrates share among themselves the highest morphological resemblance not as adults but at their "phylotypic stage" of embryonic development, as mirrored by similarity across transcriptomes. Identification of conserved brain subdivisions, established by conserved signaling centers and uniquely defined by the combinatorial expression of transcription factors during development, demonstrates that all of the general brain regions found in mammals, including the cerebral cortex, have homologies in reptiles. For example, expression of transcription factors such as Emx1 and Tbr1 in developing frogs, turtles, chickens, and mice reveals the existence of the same fundamental subdivisions of the pallium (ventral, medial, dorsal and lateral) despite the divergent morphologies of pallial structures in adults. To be clear, the cerebral cortex is thus not a mammalian invention but rather an ancient dorsal pallial structure that predates the split between sauropsids and therapsids (the mammals' precursors) (Fournier et al. 2015; Naumann et al. 2015).

Comparative studies of brain structure and development have revealed a general bauplan that describes the fundamental large-scale architecture of the vertebrate brain and provides insight into its basic functional organization. The telencephalon not only integrates and stores multimodal information but is also the higher center of action selection and motor control (basal ganglia). The hypothalamus is a conserved area controlling homeostasis and behaviors essential for survival, such as feeding and reproduction. Furthermore, in all vertebrates, behavioral states are controlled by common brainstem neuromodulatory circuits, such as the serotoneric system. Finally, vertebrates harbor a diverse set of sense organs, and their brains share pathways for processing incoming sensory inputs. For example, in all vertebrates, visual information from the retina is relayed and processed to the pallium through the tectum and the thalamus, whereas olfactory input from the nose first reaches the olfactory bulb $(\mathrm{OB})$ and then the pallium.

Although pallial structures exist in amphibians and fish, reptiles and mammals are the only vertebrates to possess a cerebral cortex with a clear, though simple, three-layered structure similar to that of mammalian allocortex. The reptilian ventral pallium also gives rise to the dorsal ventricular ridge (DVR), a structure that dominates the bird pallium and contributes to the complex cognitive abilities of birds, but whose mammalian equivalent is still the subject of debate among comparative anatomists. The reptilian cortex contains far fewer subdivisions than that of rodents, carnivores, or primates: it is subdivided into a medial cortex, often called hippocampus by anatomists, a lateral cortex, equivalent to the mammalian piriform cortex, and a dorsal cortex in between, which receives multimodal inputs (e.g., visual in turtles). There is little evidence for motor and somatosensory areas in the reptilian cortex, but pallial motor control may have evolved early in vertebrate evolution. Owing to this simplicity, the reptilian brain facilitates the study of 
primordial cortical function as a whole and points to the origins of cortex as fulfilling general associative functions.

Besides sharing pallial modules, mammals and reptiles also share a complement of cortical cell types, suggesting that some structural elements of cortical circuits arose early in amniote evolution. As in mammals, reptilian cortex contains excitatory, glutamatergic neurons and inhibitory, GABAergic interneurons. In both mammals and reptiles, these neurons have a common developmental origin: excitatory neurons are generated by multipotent cortical progenitors, whereas inhibitory neurons are born in the subpallium before migrating to the cortex.

Classical studies suggest that the reptilian main cortical cell layer (layer 2) corresponds to the deep, output layers of mammalian neocortex, whereas its layer 1 is equivalent to mammalian layer I. According to this view, mammalian cortical evolution would have included the incorporation of new, intermediate cell layers acting as input stations and internal circuitry. Morphologically, L2 pyramidal neurons of the reptilian dorsal cortex are most similar to mammalian hippocampal excitatory neurons. Indeed, reptilian pyramidal neurons have, depending on the area, little to no basal dendritic field and several densely spine-studded apical dendrites, quite different from the single, long, apical dendrite of neocortical pyramidal neurons. Consistent with this correspondence between layers, reptilian subpallial cells transplanted into mammalian embryos generate GABAergic neurons that can populate only the deeper cortical layers.

Challenging this view, however, recent molecular studies show that turtle and lizard cortical neuroblasts generate neurons that express upper layer molecular markers in a developmental sequence similar to that observed in mammals. Although the molecular characterization of neuronal types in the reptilian cortex is still in its infancy, it is possible that reptilian cortex represents an ancestral blueprint for the more elaborate mammalian cortical circuits. For example, reptilian cortical neurons, or subsets of them, might share molecular (and functional) features with both upper- and lower-layer mammalian cells.

\section{Basic Architecture and Functional Features}

\section{Vertical Connectivity}

The architecture of PCx and DCx is archetypal of a three-layered paleocortex. Layer 1 contains mainly dendrites of layer 2 principal cells, a few scattered interneurons and afferent and local axons. Layer 2 contains the densely packed somata of pyramidal cells, whose apical dendrites run radially towards the pial surface. Layer 3 contains basal dendrites of pyramidal cells, corticofugal and local axons, some interneurons and a few deep pyramidal neurons in PCx (Neville and Haberly 2004; Ulinski 1990). Incoming afferents to PCx run through the lateral olfactory tract (LOT; Sosulski et al. 2011); those to DCx run through the lateral 
forebrain bundle (LFB; Mulligan and Ulinski 1990). These input fibers fan out below the pial surface and make en-passant synapses on cortical neurons within the distal 50-100 $\mu \mathrm{m}$ of layer 1 (Haberly and Behan 1983; Smith et al. 1980). Afferent synapses impinge on both layer-1 interneurons and on distal dendrites of layer-2 pyramidal cells; interneurons provide both feed-forward and feedback inhibition to pyramidal cells that themselves provide recurrent excitation to other pyramidal neurons (Smith et al. 1980; Suzuki and Bekkers 2011, 2012; Kriegstein and Connors 1986; Mancilla et al. 1998). In both PCx and DCx, superficial layer-1 interneurons tend to receive a higher density of afferent input than pyramidal cells do (Smith et al. 1980; Suzuki and Bekkers 2012; Stokes and Isaacson 2010), which, combined with a strong feed-back inhibition via layer-2/3 interneurons (Suzuki and Bekkers 2012; Kriegstein and Connors 1986; Stokes and Isaacson 2010) may explain the observed strong inhibition evoked by sensory stimulation and the sparseness of pyramidal cell firing. To a first degree, $\mathrm{PCx}$ and $\mathrm{DCx}$ thus have a similar microcircuit layout: both exhibit distal dendritic excitation from sensory afferents, strong feed-forward inhibition, recurrent excitation through the so-called associational intracortical connections, and feedback inhibition (Haberly 2001; Shepherd 2011).

Different cell types have been identified in PCx. Most segregate into specific sub-layers of the piriform microcircuit. Excitatory neurons in layer 2 can be subdivided in semilunar (upper layer 2) and superficial pyramidal neurons (lower layer 2), whereas those in layer 3 comprise a few deep pyramidal cells and scattered multipolar spiny glutamatergic neurons (Haberly 1983; Suzuki and Bekkers 2006; Bekkers and Suzuki 2013). Although they are embedded in the same basic connectivity scheme, semilunar and superficial pyramidal cells receive different ratios of afferent to associational inputs and may therefore belong to distinct functional sub-circuits (Suzuki and Bekkers 2011; but see Poo and Isaacson 2011), consistent with morphological differences between their dendritic trees and their laminar position (Wiegand et al. 2011). Although data on subpopulations of principal cells in DCx are few, analysis of Golgi-stained material also revealed different morphological classes of spiny neurons at different laminar and sublaminar positions in reptilian cortex (Ulinski 1977; Desan 1984) PCx and DCx pyramidal neurons are also similar with respect to their dendritic electrophysiological properties, suggesting comparable integrative properties at the subcellular level (Larkum et al. 2008; Bathellier et al. 2009). Different subtypes of inhibitory interneurons have been identified in PCx based on molecular markers, the morphology of their dendritic arbor and the distribution of their axonal projections (reviewed in Suzuki and Bekkers 2007). These sub-classes seem to correlate with the type of inhibition they subserve, i.e., primarily feedback or feed-forward. Horizontal and neurogliaform interneurons in layer 1 receive afferent inputs from the LOT and mediate fast feed-forward inhibition targeting apical dendrites of layer-2 pyramidal cells. Bitufted, fast-spiking and regular spiking interneurons from layers 2 and 3 receive very little direct afferent input from the LOT but provide strong feedback inhibition onto the somata and basal dendrites of pyramidal cells (Suzuki and Bekkers 2012; Stokes and Isaacson 2010). Similarly, different 
populations of inhibitory interneurons in turtle DCx subserve mainly feed-forward (subpial cells; Mancilla et al. 1998) or feedback (Mancilla et al. 1998; Connors and Kriegstein 1986) inhibition. Axonal reconstructions of DCx interneurons (Colombe et al. 2004) and immunocytochemical labeling (Reiner 1991, 1993) suggest the existence of morphologically and physiologically identifiable classes of inhibitory interneurons. It remains to be shown that those groupings also share functional similarities with those in PCx. Given the anatomical similarity of input projections to PCx and DCx, one may speculate that the inhibitory circuit topology of these two cortices could also be similar.

\section{Horizontal Connectivity}

In PCx, afferents from mitral/tufted (MT) cells appear to project throughout the cortex without any clear topographical relationship to their glomeruli of origin (Sosulski et al. 2011; Miyamichi et al. 2011; Illig and Haberly 2003; Apicella et al. 2010; Ghosh et al. 2011). Although this does not rule out the possibility of some fine-scale topographical mapping of OB projections (e.g., mitral vs. tufted cell projections) (Igarashi et al. 2012), it is now accepted that the glomerular clustering of olfactory receptor cells axons in OB is entirely discarded at the level of PCx (Wilson and Sullivan 2011). In DCx, early tracing studies from Ulinski and colleagues suggested that the visual field is projected onto the rostro-caudal axis of DCx in the form of iso-azimuth lamellae covering the naso-temporal dimension of the visual field (Mulligan and Ulinski 1990; Ulinski and Nautiyal 1988). Such a mapping of projections still awaits physiological confirmation and fine thalamocortical projection tracing. If confirmed, this topographical mapping would differ from the topology of mammalian olfactory projections to $\mathrm{PCx}$, at least along one cortical dimension.

In both PCx and DCx, the density of sensory afferents varies over the cortical surface: high rostrally and laterally, it decreases progressively as one moves away from the entry point of the LOT (PCx) or the LFB (DCx). Hence, the balance between afferent and associational connectivity decreases along the rostro-caudal and latero-medial (or ventro-dorsal) axes (Mulligan and Ulinski 1990; Haberly 2001; Wilson and Sullivan 2011; Hagiwara et al. 2012; Cosans and Ulinski 1990). PCx is subdivided into anterior and posterior regions, which differ not only in the density of afferent vs. associational fibers (Haberly 2001) but also in the properties of odor-evoked responses (Litaudon et al. 2003; Kadohisa and Wilson 2006). PCx microcircuits may also contain fine-grain connectivity gradients: in vitro recordings from aPCx reveal that inhibition of pyramidal cells is asymmetric and stronger along the rostro-caudal axis of the anterior part of PCx, over distances as short as $200 \mu \mathrm{m}$ (Luna and Pettit 2010). In turtles, DCx has been classically divided into two different regions (D2 and D1) along the latero-medial axis (Ulinski 1990; Desan 1984). This dichotomy rests mostly on cytoarchitectural features related to the thickness of subcellular layer 3: thick in D2 laterally, thin in 
D1, with a significant transition zone between the two. Recent molecular data suggest that this separation may be correlated with a higher expression level of layer-4 markers in D2 (Dugas-ford et al. 2012). Confirmation of this division and of its potential functional significance needs additional work. Such gradients of connectivity across the cortical surface (in PCx and DCx) should be clearly described because any horizontal heterogeneity could influence the propagation and reverberation of activity across cortex, under the combined influences of spreading afferent input and widespread associational activity.

Given their reciprocal interconnections with high-order cortical areas and a lack of evident sensory topography, PCx and DCx are sometime described as associational rather than primary sensory cortices (Shepherd 2011). The major partners of PCx are the orbitofrontal cortex (Ekstrand et al. 2001; Illig 2006), the lateral entorhinal cortex (Kerr et al. 2007; Johnson et al. 2000) and the agranular insular cortex (Johnson et al. 2000). Connectivity to these downstream targets differs between aPCx and pPCx, supporting the notion that they have different functions. Similarly, DCx is reciprocally connected to dorso-medial (DMCx) and medial (MCx) cortices (Ulinski 1977; Desan 1984). Those regions are, on the basis of hodology and position, often compared to parahippocampal and hippocampal cortices (Desan 1984; Northcutt 1981; López et al. 2003; Aboitiz et al. 2003). Both PCx and DCx are thus directly connected to associational networks likely involved in controlling or modulating behavior.

PCx and DCx are further interconnected with other cortical-like areas that also receive parallel sensory afferents from the $\mathrm{OB}$ or the lateral geniculate nucleus of the thalamus (LGN), respectively. For PCx, these include the anterior olfactory nucleus (AON; Haberly and Price 1978; Illig and Eudy 2009), the olfactory tubercule (OT; Haberly and Price 1978), and the amygdala (Johnson et al. 2000; Luna and Morozov 2012). AON might be a first stage of odorant-feature processing, in turn used by PCx to detect complex odorant combinations (Haberly 2001; Lei et al. 2006; Kay et al. 2011). DCx's AON equivalent could be the pallial thickening (PT), for it receives direct thalamic afferent input and projects to DCx (Mulligan and Ulinski 1990; Heller and Ulinski 1987). If AON and PT also share functional characteristics, these similarities may point to common elementary processing streams of three-layered sensory cortices.

In turtles, visual stimulation triggers propagating waves of neural activity that travel across the cortex. These waves are slower and simpler than those observed in mammalian neocortex. They are accompanied by relatively slow oscillations, which are most prominent in the $20 \mathrm{~Hz}$ frequency band. Whereas the so-called gamma oscillations in mammalian cortex are typically around and above $40 \mathrm{~Hz}$, recent results in mice indicate that the $20 \mathrm{~Hz}$ band dominates when parvalbumin (PV) interneuron development is artificially arrested, consistent with the above observation that turtle cortex lacks PV interneurons. The computational role, if any, of such dynamics is unknown at present. Progress will require new experimental approaches that allow the simultaneous sampling of large neuronal populations. Specific and data-driven theories of computation in reptilian cortex thus await further study. To the extent that modern reptilian cortex resembles that in the 
common ancestor of reptiles and mammals, understanding reptilian cortex function may reveal some of the fundamental associative computations that early cortical circuits evolved to carry out.

\section{Physiological Adaptations of the Reptilian Brain}

Reptiles are ectotherms and must behaviorally or physiologically adapt to varying temperatures, making reptile brains notable for their adaptation to extreme conditions. The turtle brain, for example, has evolved remarkable adaptations to hypoxic conditions, which have long been known to experimentalists interested in hearing, olfaction, motor control or cerebellar physiology. Semi-aquatic freshwater turtles (e.g., Chrysemys picta) are remarkable for their ability to survive hibernation in hypoxic waters during cold northern winters and have been reported to survive anoxic periods of over 4 months at $3{ }^{\circ} \mathrm{C}$. This resilience points to several physiological adaptations to survive cold, anoxic conditions during hibernation and subsequent re-oxygenation in the spring. The mechanisms underlying such tolerance are interesting — and also possibly applicable to the treatment of brain injury due to ischemia.

Anoxia tolerance critically depends on the ability to reduce energy expenditure while elevating anaerobic adenosine triphosphate (ATP) production. ATP consumption in neural tissues, particularly those involved in sensory functions, requires high ATP turnover. Free glucose and glycogen can serve as metabolic substrates in anoxic conditions, and stores of glycogen are released from the liver and skeletal muscles to facilitate ATP production during hibernation. But by shifting to anaerobic glycolysis, hibernating turtles must buffer the resulting metabolic product, lactic acid, to prevent fatal acidosis. In turtles, extracellular buffering of lactic acid is achieved by the large volumes of pericardial and peritoneal fluids and through sequestration in the mineralized shell and skeleton.

During hibernation, turtles reduce ATP consumption in neural tissue by reducing membrane permeability and, thus, the need for pump-mediated restoration of ionic balance. This phenomenon has been observed in turtle neurons for voltage-gated $\mathrm{Na}^{+}$channels, the oxygen-sensitive calcium-activated $\mathrm{K}^{+}$channels, as well as AMPA and NMDA receptors. Neuronal depolarization and NMDA receptor over-activation are hallmarks of anoxia in mammalian neural tissue and lead to excitotoxic cell death via increased intracellular $\mathrm{Ca}^{2+}$ concentrations. In turtle neurons, NMDA receptor opening times are reduced by $65 \%$ after $60 \mathrm{~min}$ of anoxia, and NMDA receptor currents are actually silenced by anoxia-triggered cytosolic $\mathrm{Ca}^{2+}$ release from mitochondrial stores. Despite reduced channel activity and, thus, reduced electrical activity during anoxia, turtles appear to remain vigilant and responsive to visual stimuli during hibernation (but less so to tactile stimuli). This maintenance of visual responses in the absence of tactile responses during hibernation suggests a differential down-regulation of sensory modalities. Because changes in daily illumination contribute to signaling the end of hibernation, it may 
be a selective advantage for energy-conserving mechanisms to be applied less to the visual system than to other brain areas.

When oxygen again becomes available, the re-oxygenation of tissue, such as that observed after ischemia-reperfusion events caused by myocardial infarction or stroke, is problematic in mammals because it is associated with the excessive production of reactive oxygen species (ROS) that damage cells through protein oxidation and lipid peroxidation. Turtles have evolved effective physiological mechanisms that allow for repeated cycles of anoxia and re-oxygenation without the free-radical damage induced by ischemia-reperfusion of tissues observed in mammals. These mechanisms involve an evolutionarily old up-regulation of nitric oxide and its metabolites that limits ROS generation and tissue damage in cardiac and brain tissue. Thus, the turtle brain, with these physiological adaptations, is a very useful model for the study of neural circuit function. Neural tissue remains alive and viable for days ex vivo, and tissue can be routinely stored overnight in a refrigerator at $3-5^{\circ} \mathrm{C}$.

\section{Brain and Behavior}

Reptiles express a number of complex behaviors normally attributed to mammals. They can, for example, learn to navigate mazes as well as birds or mammals and likely use a hippocampal structure to do so. Remarkably, sea turtles navigate across the ocean to their home beach, possibly using magnetic, olfactory, and visual cues. Reptiles also express interesting social behaviors: pythons and crocodiles, for example, incubate their eggs after hatching, and mothers guard the nest and provide infant care. Some lizard species exhibit pair bonding and parental care and form families. Others construct networks of tunnels and may live in social communities like those of naked-mole rats.

Reptiles inhabit a variety of ecological niches, such as terrestrial deserts, temperate forests, tropical jungles, the sea, and fresh waters. They are found in all oceans and on all continents except Antarctica. Semi-aquatic turtles, which live in primarily aquatic habitats but make terrestrial excursions to lay eggs, offer an interesting model animal in which to study navigation. Many turtles are known for their impressive navigational skills and high nest-site fidelity, or the tendency for individuals to return to the same geographic location or microhabitat to nest. The mechanisms underlying these behaviors are not entirely understood, but navigational ability in the fresh water turtle Chrysemys picta seems to depend on experience gained during a critical period ( $<4$ years of age). Translocated resident adults, for example, can quickly and consistently find new aquatic habitats within $33 \mathrm{~h}$, often using precise and predictable routes, whereas translocated non-resident adults appear unable to find new aquatic habitats even after 21 days.

The hippocampus is critical for map-like or relational memory representations of allocentric space, where objects are represented in relation to other objects. In birds and mammals, lesions to the hippocampal formation produce selective impairments 
in spatial tasks that require the encoding of relationships among multiple environmental features (place learning) but not in tasks that require approaching a single cue or simple non-spatial discriminations. Whereas extensive comparative research supports the idea that the reptilian medial cortex is homologous to the hippocampal formation of mammals and birds, only a few studies have examined the neural function of this brain structure or its role in place learning. In one such study, Rodríguez et al. (2002) evaluated the effects of lesions to the hippocampus of turtles in place and cue-maze tasks. Hippocampus-lesioned (and sham-lesioned) animals performed cue-discrimination tasks correctly but hippocampus-lesioned animals failed at the place learning that relied on allocentric space learning. These results indicate that lesions to the hippocampus of turtles selectively impair map-like memory representations of the environmental space, mirroring the effect of hippocampal lesions in mammals and birds. Thus reptilian hippocampus may also share a central role in navigation.

In conclusion, the observation that mammalian and reptilian brains share both ancestry and a large number of functional attributes suggests that the identification of primordial (and possibly general) algorithmic principles of brain function could be helped by comparative approaches. To this end, the reptilian brain, with its simpler structure, may prove invaluable to decipher fundamental questions of modern neuroscience.

Open Access This chapter is distributed under the terms of the Creative Commons AttributionNoncommercial 2.5 License (http://creativecommons.org/licenses/by-nc/2.5/) which permits any noncommercial use, distribution, and reproduction in any medium, provided the original author(s) and source are credited.

The images or other third party material in this chapter are included in the work's Creative Commons license, unless indicated otherwise in the credit line; if such material is not included in the work's Creative Commons license and the respective action is not permitted by statutory regulation, users will need to obtain permission from the license holder to duplicate, adapt or reproduce the material.

\section{References}

Aboitiz F, Morales D, Montiel J (2003) The evolutionary origin of the mammalian isocortex: towards an integrated developmental and functional approach. Behav Brain Sci 26:535-552

Apicella A, Yuan Q, Scanziani M, Isaacson JS (2010) Pyramidal cells in piriform cortex receive convergent input from distinct olfactory bulb glomeruli. J Neurosci 30:14255-14260

Bathellier B, Margrie TW, Larkum ME (2009) Properties of piriform cortex pyramidal cell dendrites: implications for olfactory circuit design. J Neurosci 29:12641-12652

Bekkers JM, Suzuki N (2013) Neurons and circuits for odor processing in the piriform cortex. Trends Neurosci 36:429-438

Colombe JB, Sylvester J, Block J (2004) Subpial and stellate cells: two populations of interneurons in turtle visual cortex. J Comp Neurol 471:333-351

Connors BW, Kriegstein AFL (1986) Cellular physiology of the turtle visual cortex: distinctive properties of pyramidal and stellate neurons. J Neurosci 6:164-177 
Cosans CE, Ulinski PS (1990) Spatial organization of axons in turtle visual cortex: intralamellar and interlamellar projections. J Comp Neurol 296:548-558

Desan PH (1984) The organization of the cerebral cortex of the pond turtle, Pseudemys scripta elegans. PhD thesis, Harvard University, Cambridge, MA

Dugas-ford J, Rowell JJ, Ragsdale CW (2012) Cell-type homologies and the origins of the neocortex. Proc Natl Acad Sci USA 109:16974-16979

Ekstrand JJ, Domroese ME, Johnson DMG, Feig SL, Knodel SM, Behan M, Haberly LB (2001) A new subdivision of anterior piriform cortex and associated deep nucleus with novel features of interest for olfaction and epilepsy. J Comp Neurol 434:289-307

Fournier J, Müller CM, Laurent G (2015) Looking for the roots of cortical sensory computation in three-layered cortices. Curr Opin Neurobiol 31:119-126

Ghosh S, Larson SD, Hefzi H, Marnoy Z, Cutforth T, Dokka K, Baldwin KK (2011) Sensory maps in the olfactory cortex defined by long-range viral tracing of single neurons. Nature 472:217-220

Haberly LB (1983) Structure of the piriform cortex of the opossum. I. Description of neuron types with Golgi methods. J Comp Neurol 213:163-187

Haberly LB (2001) Parallel-distributed processing in olfactory cortex: new insights from morphological and physiological analysis of neuronal circuitry. Chem Senses 26:551-576

Haberly L, Behan M (1983) Structure of the piriform cortex of the opossum. III. Ultrastructural characterization of synaptic terminals of association and olfactory bulb afferent fibers. J Comp Neurol 219:448-460

Haberly LB, Price JL (1978) Association and commissural fiber systems of the olfactory cortex of the rat. J Comp Neurol 178:711-740

Hagiwara A, Pal SK, Sato TF, Wienisch M, Murthy VN, Shepherd GM (2012) Optophysiological analysis of associational circuits in the olfactory cortex. Front Neural Circ 6:18

Heller SB, Ulinski PS (1987) Morphology of geniculocortical axons in turtles of the genera Pseudemys and Chrysemys. Anat Embryol 175:505-515

Igarashi KM, Ieki N, An M, Yamaguchi Y, Nagayama S, Kobayakawa K, Kobayakawa R, Tanifuji M, Sakano H, Chen WR et al (2012) Parallel mitral and tufted cell pathways route distinct odor information to different targets in the olfactory cortex. J Neurosci 32:7970-7985

Illig KR (2006) Projections from orbitofrontal cortex to anterior piriform cortex in the rat suggest a role in olfactory information processing. J Comp Neurol 488:224-231

Illig KR, Eudy JD (2009) Contralateral projections of the rat anterior olfactory nucleus. J Comp Neurol 512:115-123

Illig KR, Haberly LB (2003) Odor-evoked activity is spatially distributed in piriform cortex. J Comp Neurol 457:361-373

Johnson DMG, Illig KR, Behan M, Haberly LB (2000) New features of connectivity in piriform cortex visualized by intracellular injection of pyramidal cells suggest that "primary" olfactory cortex functions like "association" cortex in other sensory systems. J Neurosci 20:6974-6982

Kadohisa M, Wilson DA (2006) Separate encoding of identity and similarity of complex familiar odors in piriform cortex. Proc Natl Acad Sci USA 103:15206-15211

Kay RB, Meyer EA, Illig KR, Brunjes PC (2011) Spatial distribution of neural activity in the anterior olfactory nucleus evoked by odor and electrical stimulation. J Comp Neurol 519:277-289

Kerr KM, Agster KL, Furtak SC, Burwell RD (2007) Functional neuroanatomy of the parahippocampal region: the lateral and medial entorhinal areas. Hippocampus 17:697-708

Kriegstein R, Connors BW (1986) Cellular physiology of the turtle visual cortex: synaptic properties and intrinsic circuitry. J Neurosci 6:178-191

Larkum ME, Watanabe S, Lasser-ross N, Rhodes P, Ross WN, Ledergerber D, Larkum ME (2008) Dendritic properties of turtle pyramidal neurons. J Neurophysiol 99:683-694

Lei H, Mooney R, Katz LC (2006) Synaptic integration of olfactory information in mouse anterior olfactory nucleus. J Neurosci 26:12023-12032

Litaudon P, Amat C, Bertrand B, Vigouroux M, Buonviso N (2003) Piriform cortex functional heterogeneity revealed by cellular responses to odours. Eur J Neurosci 17:2457-2461 
López JC, Vargas JP, Gómez Y, Salas C (2003) Spatial and non-spatial learning in turtles: the role of medial cortex. Behav Brain Res 143:109-120

Luna VM, Morozov A (2012) Input-specific excitation of olfactory cortex microcircuits. Front Neural Circ 6:1-7

Luna VM, Pettit DL (2010) Asymmetric rostro-caudal inhibition in the primary olfactory cortex. Nat Neurosci 13:533-535

Mancilla JG, Fowler M, Ulinski PS (1998) Responses of regular spiking and fast spiking cells in turtle visual cortex to light flashes. Vis Neurosci 15:979-993

Miyamichi K, Amat F, Moussavi F, Wang C, Wickersham I, Wall NR, Taniguchi H, Tasic B, Huang ZJ, He Z et al (2011) Cortical representations of olfactory input by trans-synaptic tracing. Nature 472:191-196

Mulligan KA, Ulinski PS (1990) Organization of geniculocortical projections in turtles: isoazimuth lamellae in the visual cortex. J Comp Neurol 296:531-547

Naumann R, Ondracek JM, Reiter S, Shein-Idelson M, Tosches MA, Yamawaki T, Laurent G (2015) Reptilian brain primer. Curr Biol 25(8):R317-R321

Neville KR, Haberly LB (2004) Olfactory cortex. In: Shepherd GM (ed) The synaptic organization of the brain. Oxford University Press, New York, NY, pp 415-454

Northcutt RG (1981) Evolution of the telencephalon in nonmammals. Annu Rev Neurosci 4:301-350

Poo C, Isaacson JS (2011) A major role for intracortical circuits in the strength and tuning of odorevoked excitation in olfactory cortex. Neuron 72:41-48

Reiner A (1991) A comparison of the neurotransmitter-specific and neuropeptide-specific neuronal cell types present in turtle cortex to those present in mammalian isocortex: implications for the evolution of isocortex. Brain Behav Evol 38:53-91

Reiner A (1993) Neurotransmitter organization and connections of turtle cortex: implications for the evolution of mammalian isocortex. Comp Biochem Physiol 104:735-748

Rodríguez F, López JC, Vargas JP, Gómez Y, Broglio C, Salas C (2002) Conservation of spatial memory function in the pallial forebrain of reptiles and ray-finned fishes. J Neurosci 22 (7):2894-2903

Shepherd GM (2011) The microcircuit concept applied to cortical evolution: from three-layer to six-layer cortex. Front Neuroanat 5:1-15

Smith LM, Ebner FF, Colonnier M (1980) The thalamocortical projection in Pseudemys turtles: a quantitative electron microscopic study. J Comp Neurol 461:445-461

Sosulski DL, Bloom ML, Cutforth T, Axel R, Datta SR (2011) Distinct representations of olfactory information in different cortical centres. Nature 472:213-216

Stokes CCA, Isaacson JS (2010) From dendrite to soma: dynamic routing of inhibition by complementary interneuron microcircuits in olfactory cortex. Neuron 67:452-465

Suzuki N, Bekkers JM (2006) Neural coding by two classes of principal cells in the mouse piriform cortex. J Neurosci 26:11938-11947

Suzuki N, Bekkers JM (2007) Inhibitory interneurons in the piriform cortex. Clin Exp Pharmacol Physiol 34:1064-1069

Suzuki N, Bekkers JM (2011) Two layers of synaptic processing by principal neurons in piriform cortex. J Neurosci 31:2156-2166

Suzuki N, Bekkers JM (2012) Microcircuits mediating feedforward and feedback synaptic inhibition in the piriform cortex. J Neurosci 32:919-931

Ulinski PS (1977) Intrinsic organization of snake medial cortex: an electron microscopic and Golgi study. J Morphol 152:247-279

Ulinski PS (1990) The cerebral cortex of reptiles. Cereb Cort 8A:139-216

Ulinski PS, Nautiyal J (1988) Organization of retinogeniculate projections in turtles of the genera Pseudemys and Chrysemys. J Comp Neurol 276:92-112

Wiegand HF, Beed P, Bendels MHK, Leibold C, Schmitz D, Johenning FW (2011) Complementary sensory and associative microcircuitry in primary olfactory cortex. J Neurosci 31:12149-12158

Wilson DA, Sullivan RM (2011) Cortical processing of odor objects. Neuron 72:506-519 